\title{
The Effect of Hippocampal Cognitive Impairment and XIAP on Glucose and Lipids Metabolism in Rats
}

\author{
Chunbo Xia ${ }^{a}$ Lin Zhu ${ }^{a}$ Wenhua Shaob Sisi Mib Sen Du ${ }^{a}$ Lin Yea Manjun Liuc \\ YongJun Pang $^{d}$ Linlin Nonge Changwen Jiang ${ }^{a}$ Hailu Zhao ${ }^{f}$ Guangying Qi ${ }^{b}$ \\ aDepartment of Anthropotomy, Guilin Medical University, Guilin, 'bepartment of Physiopathology, \\ Guilin Medical University, Guilin, 'Department of Pediatrics, Guilin Medical University, Affiliated \\ Hospital, Guilin, dDepartment of Physiology, Guilin Medical University, Guilin, eDepartment of Histology \\ and Embryology, Guilin Medical University, Guilin, 'DDepartment of Immunology, Guilin Medical \\ University, Guilin, P.R. China
}

\section{Key Words}

Glucose and lipids metabolism - Cognitive disorders - Hippocampus • Neuron apoptosis • XIAP • Gastrointestinal motility

\begin{abstract}
Background/Aims: To investigate the effect of cognitive impairment and X-linked inhibitor of apoptosis protein (XIAP) on glucolipid metabolism. Materials and Methods: $\beta$-amyloid (A $\beta$ 1-42) was injected into the hippocampus of rats to establish a cognitive impairment model. Trans-activator of transcription (TAT)-XIAP fusion protein (the TAT-XIAP group), PBS (the model group), or XIAP antisense oligonucleotides (the ASODN group) was injected into the lateral ventricles of the rats to increase and decrease the activity of XIAP in the hippocampus. To determine the level of blood glucose and lipids, adenosine monophosphate-activated protein kinase (AMPK) expression of liver and hipppocamual neuronal apoptosis. Results: The levels of FPG, TG, TC and LDL were significantly higher in the TAT-XIAP group, the model group and the ASODN group than in the blank group $(P<0.05)$; however, the HDL level showed no significant change in all groups of rats. The apoptosis indexes of the rat hippocampal CA1 neuron were $68.44 \pm 4.31 \%, 13.21 \pm 2.30 \%, 56.68 \pm 4.771 \%$, and $87.51 \pm 6.63 \%$ in the model group, the blank group, the TAT-XIAP group and the ASODN group, respectively. Gastrointestinal motility was less frequent (per time unit) in the model group, the ASODN group and the TAT-XIAP group than in the blank group. Compared with the model group, gastrointestinal motility was significantly less frequent in the ASODN group and was significantly more frequent in the TAT-XIAP group. Compared with the blank group, the model group had a significantly lower gastric emptying rate and intestinal propulsive rate. Compared with the model group, the gastric emptying rate and intestinal propulsive rate were significantly lower in the ASODN group and were significantly higher in the TAT-XIAP group. Compared with the blank group,

C. Xia and L. Zhu contributed equally to this article.

Dr Chunbo Xia and Dr Guangying Qi

Department of Anthropotomy, Guilin Medical University, Guilin 541004, (P.R. China); and Department of Physiopathology, Guilin Medical University, 109, Huancheng North 2 Road, Guilin 541004, Qixing District (P.R. China)

Tel.+86-733-589-5385, E-Mail xiachunbo910@163.com and 2608689827@qq.com
\end{abstract}

KARGER 


\section{Cellular Physiology Cell Physiol Biochem 2016;38:609-618 \begin{tabular}{ll|l} 
and Biochemistry Published online: February 05, 2016 & $\begin{array}{l}\text { (c) } 2016 \text { The Author(s). Published by S. Karger AG, Basel } \\
\text { www.karger.com/cpb }\end{array}$ \\
\hline
\end{tabular} \\ Xia et al.: The Effect of Hippocampal Cognitive Impairment}

the expressions of AMPK mRNA, and AMPK protein were significantly reduced in the model group, the TAT-XIAP group, and the ASODN group. AMPK expression was significantly increased in the TAT-XIAP group and was significantly decreased in the ASODN group than in the model group. Conclusion: Cognitive impairment and hippocampal neuron apoptosis can cause glucose and lipids metabolic abnormalities, possibly by regulating gastrointestinal motility and AMPK expression in the liver. The changes in the function of XIAP, which is an anti-apoptotic protein in the hippocampus, may affect the metabolism of glucose and lipids.

\section{Introduction}

(C) 2016 The Author(s)

Published by S. Karger AG, Basel

Diabetes and Alzheimer's disease (AD) are a group of complex, interrelated diseases. Several studies have shown that $60 \%-70 \%$ of diabetic patients have varying degrees of cognitive impairment, which is mainly manifested as reduced learning and memory abilities, and a significantly increased risk of dementia $[1,2]$. Up to $81 \%$ of $A D$ patients have varying degrees of abnormal glucose metabolism [3]. Diabetes and AD share many pathophysiological factors, such as an abnormal insulin signaling pathway, an increase in advanced glycation end products (AGEs), oxidative stress, and inflammation reactions, that may be involved in disease development; however, the exact mechanisms are still unknown. Due to its close relationship to diabetes, $\mathrm{AD}$ is also known as type 3 diabetes. Anatomically, the hippocampus is part of the cortex structure of the limbic forebrain, and its relation to cognitive function has become a popular research topic $[1,2]$. The hippocampus, which is the visceral integration hub, can also regulate visceral activities through autonomic pathways. The hippocampus receives the visceral afferent nerve fibers and has extensive fiber connections with the part of the central nervous system that regulates visceral activities, such as the hypothalamus, brain stem and spinal cord. The hippocampus regulates visceral functions through sympathetic and parasympathetic nerves. In clinical practice, AD patients often have abnormal levels of blood glucose and lipids [3], and hippocampal neuron apoptosis is the pathological characteristic and outcome of the hippocampus in AD patients[1,2, 4, 5]. Despite the similarities between diabetes and $\mathrm{AD}$, whether hippocampal neuron apoptosis affects the levels of peripheral blood glucose and lipids and the underlying mechanisms are still unknown. This study aims to develop an in-depth understanding of the effect and mechanism of hippocampal cognitive impairment on the metabolism of blood glucose and lipids. $\beta$-amyloid (A $\beta$ 1-42) was injected into the hippocampus of rats to establish a cognitive impairment model, and changes in blood glucose and lipid levels were observed by increasing and decreasing the activity of XIAP in the hippocampus. The relationship between the changes in blood glucose and lipid levels and hippocampal neuron apoptosis, the expression of AMPK, and gastrointestinal motility was analyzed.

\section{Materials and Methods}

Main instruments and laboratory reagents

A stereotaxic instrument (SR-6, Japan), water maze (Anhui Zhenghua Biological Instrument Equipment Co., Ltd., China), automatic biochemical analyzer (Erba XL-600, Germany), B-mode ultrasonography machine (IE33 Philips, with L11-3 probe), PCR instrument (T100, BIO-RAD, USA), and gel imaging system (BIO-RAD, USA) were utilized in this study.

A $31-42$ was purchased from Sigma Incorporation. Isopropyl-B-D-thiogalactoside (IPTG), urea, and imidazole were purchased from Amresco LLC. Glucose and lipids assay kits were purchased from Youlite Medical Treatment Electron (Group) Limited (Guilin, China). The terminal deoxynucleotidyl transferase dUTP nick end labeling (TUNEL) (POD) assay (in situ apoptosis detection kit) was purchased from HoffmannLa Roche AG (USA). The pTAT-HA plasmid was kindly provided by Steven F. Dowdy (USA). The nitrilotriacetic acid (Ni-NTA) ion affinity chromatography kit was purchased from Bio Basic Inc. Horseradish peroxidase 


\section{Cellular Physiology Cell Physiol Biochem 2016;38:609-618

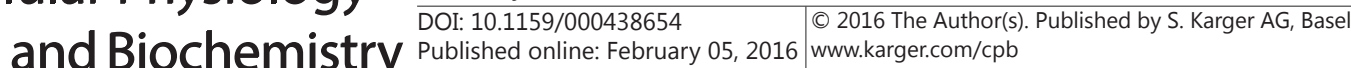 \\ Xia et al.: The Effect of Hippocampal Cognitive Impairment}

(HRP)-labeled goat anti-mouse IgG and enhanced chemiluminescence ECL (ECL) kits were purchased from Beijing Zhongshan Golden Bridge Biotechnology Co., Ltd, and the AMPK antibody was purchased from Proteintech Group.

\section{Animal model and experimental groups}

Spraque-Dawley (SD) rats (male or female, 250 - 300 g) were provided by the Experimental Animal Center of Guilin Medical College. The animals were cared for in compliance with the principles and guidelines of Ethical Committee for Animal Care and Institutional Animal Ethical Committee of Guilin Medical University, in accordance with the China National Law on Animal Care and Use. With reference to the rat brain stereotaxic coordinates, $\mathrm{A} \beta 1-42(10 \mu \mathrm{g} / \mathrm{rat})$ was injected into the rat hippocampus (3.9 $\mathrm{mm}$ posterior to the anterior fontanelle, $3.3 \mathrm{~mm}$ right of the midline, $3.1 \mathrm{~mm}$ needle depth). The water maze test was performed to screen 30 rats for cognitive impairment. The inclusion criterion was cognitive impairment as confirmed by a significant difference in the location orientation and spatial exploration of the water maze relative to the control group. The rats with cognitive impairment were then randomly assigned into one of the following groups: the TAT-XIAP group (injection of trans-activator of transcription [TAT]XIAP fusion protein into the right lateral ventricle; $80 \mathrm{mg} / \mathrm{kg}, 3$ days), the ASODN group (injection of XIAP antisense oligonucleotides into the right lateral ventricle; $2 \mathrm{mg} / \mathrm{kg}, 3$ days), and the model group (injection of an equal volume of phosphate buffered saline [PBS] into the right lateral ventricle) with 10 rats in each group. An additional 10 rats were used as the blank group (absence of any interference).

\section{Evaluation of the learning and memory abilities of rats with the water maze test}

The water maze was a round pool that was $130 \mathrm{~cm}$ in diameter, $50 \mathrm{~cm}$ tall, and $30 \mathrm{~cm}$ deep. After the water maze was filled with water, milk powder was added (approximately 1.5\%) to make the water opaque white. The water temperature was $22-25^{\circ} \mathrm{C}$. The pool was divided into quadrants $1,2,3$ and 4 clockwise. A quadrant was chosen, and a round transparent platform that was $10 \mathrm{~cm}$ in diameter and 28 $\mathrm{cm}$ high was placed in its center with the top of platform located $2 \mathrm{~cm}$ below the water surface. A camera was installed above the maze for real-time recording of the rat's movements. The external references of the maze remained constant, the curtain was closed, and the laboratory light source was kept hidden with a consistent brightness. On the day prior to the location orientation test, the rats were individually placed into the pool (no platform) to swim freely for 2-3 minutes to become familiar with the maze environment. The rats were then placed into the maze at a new randomly selected starting point (1/quadrant) while facing the maze wall, and the time from being placed into the pool until locating and climbing onto the platform was recorded for each rat; this time was called the escape latency. If the rat was unable to locate the platform after 120 seconds, it was guided towards the platform and stayed there for 10 seconds, and the escape latency was recorded as 120 seconds. For the spatial exploration test, the platform was removed, a pool entry point was randomly selected, and the rats were placed into the pool facing the maze wall. The following information was recorded: the distance that the rat swam in each quadrant, the ratio to the total swimming distance, and the number of times the rat crossed the platform during the 120 seconds.

\section{Preparation of the fusion protein expressed in the TAT-XIAP expression vector}

The full-length cDNA sequence of rat XIAP was inserted into the pTAT-HA plasmid that was digested by NcoI and XhoI to construct the pTAT-XIAP prokaryotic expression vector. The transformation was performed following the instructions for BL21plysS competent cells, and ampicillin (Amp) was used to screen positive colonies. The pTAT-XIAP positive colonies were then inoculated into Amp-containing LB broth, which was placed on a shaker at $37^{\circ}$ overnight. Active bacterial broth was then inoculated into $10 \mathrm{~mL}$ of LB broth (Amp $100 \mathrm{mg} / \mathrm{L}$ ); once OD600 reached 0.8-1, $1 \mathrm{mmol} / \mathrm{L}$ IPTG was added to induce expression at $37^{\circ}$ for 4 hours. The induced bacteria were collected by centrifugation, washed with PBS twice, resuspended with a lysis buffer, allowed to stand at $4^{\circ}$ for 30 minutes, and subjected to ultrasonication in an ice bath $(5 \mathrm{~g} / \mathrm{mL}, \mathrm{M} / \mathrm{V})$ followed by centrifugation at $12,000 \mathrm{rpm}$ at $4^{\circ}$ for 30 minutes. The precipitate was collected and successively washed with $2 \mathrm{~mol} / \mathrm{L}$ and $4 \mathrm{~mol} / \mathrm{L}$ urea. Next, $8 \mathrm{~mol} / \mathrm{L}$ urea was added to dissolve the precipitate in an ice bath for 1 hour followed by centrifugation at 12,000 rpm at $4^{\circ}$ for 20 minutes. The supernatant was collected and added to a Ni-NTA affinity chromatography column and successively eluted with $15 \mathrm{~mL}$ of $20 \mathrm{mmol} / \mathrm{L}$ and $50 \mathrm{mmol} / \mathrm{L}$ imidazole solution. Next, $100 \mathrm{mmol} / \mathrm{L}$ imidazole solution was used to collect the purified protein, which was filtered with a $0.22 \mu \mathrm{m}$ membrane. The protein samples were subjected 


\section{Cellular Physiology Cell Physiol Biochem 2016;38:609-618

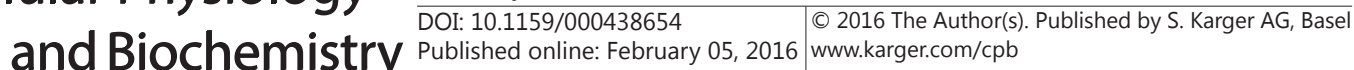 \\ Xia et al.: The Effect of Hippocampal Cognitive Impairment}

to sodium dodecyl sulfate-polyacrylamide gel electrophoresis (SDS-PAGE) and electrically transferred to a nitrocellulose membrane for western blot analysis. Mouse anti-6His monoclonal antibody was used as the primary antibody, HRP-labeled goat anti-mouse IgG was used as the secondary antibody, ECL reagents were used for chemiluminescence, and the membrane was exposed to X-rays and observed. After $10 \%$ glycerol and $0.014 \mathrm{~mol} / \mathrm{L} \beta$-mercaptoethanol were added, the purified products were successively dialyzed with 6 , $5,4,2$, and $1 \mathrm{~mol} / \mathrm{L}$ urea buffers and PBS with magnetic stirring at $4^{\circ}$ and then placed in a $-20^{\circ}$ refrigerator to freeze overnight followed by vacuum freeze-drying and storage at $-80^{\circ}$ for later use.

Determination of the levels of glucose and lipids, observation of gastrointestinal motility, and treatment of tissue samples of rats

Based on the experimental groups, TAT-XIAP fusion protein, PBS, and XIAP antisense oligonucleotides were injected into the right lateral ventricles of the successful rat models. The rats were raised for 4 additional weeks. Next, under anesthesia with chloral hydrate, blood samples were taken from the heart, and the plasma was separated and loaded into an automatic biochemical analyzer to determine the levels of fasting plasma glucose (FPG), triglyceride (TG), cholesterol (TC), high-density lipoprotein (HDL) cholesterol and low-density lipoprotein (LDL) cholesterol. B-mode ultrasonography was performed to dynamically observe the gastrointestinal motility of the rats. The method of [6] was used to determine the gastric emptying rate and intestinal propulsive rate. After fasting for 24 hours, the rats were fed a black semi-solid nutritious paste via oral gavage $(1 \mathrm{~mL} / 100 \mathrm{~g})$. The gastric emptying rate = 1 - (full stomach weight - empty stomach weight)/ gavage volume; the intestinal propulsive rate $=$ distance between pylorus to the front of the black semisolid paste/full length from the pylorus to the ileocecal junction. Thirty minutes after gavage and under anesthesia with $10 \%$ chloral hydrate $(4 \mathrm{ml} / \mathrm{kg})$, tissue from the rat's liver was collected, placed in liquid nitrogen, and stored for later use. The gastric cardia and pylorus were ligated. The gastric emptying rate and intestinal propulsive rate were calculated. The heart was perfused and fixed. The neck was broken, and the brain was removed.

\section{Detection of hippocampal neuron apoptosis in rats with the TUNEL assay}

The right hippocampus (non-injury site) was removed, embedded with paraffin, prepared into sections, rendered transparent by xylene, hydrated in gradient alcohol, incubated with proteinase $\mathrm{K}$ at $37^{\circ}$ for 20-30 minutes, immersed in 3\% H2O2 solution for 10 minutes, washed by PBS 3 times (3 minutes/ wash), incubated with $50 \mu \mathrm{L}$ TUNEL reaction mixture in a dark box at $37^{\circ}$ for 1 hour, washed by PBS 3 times ( 3 minutes/wash), incubated with $50 \mu \mathrm{L}$ converter-POD at $37^{\circ}$ for 30 minutes, washed by PBS 3 times ( 3 minutes/wash), stained with diaminobenzidine (DAB), and mounted with a neutral resin. An equal volume of labeling solution (no terminal transferase) was used in place of the TUNEL working solution as the negative control. Neurons were considered positive if brown or yellow particles were present in the nucleus. High-resolution microscopic fields were randomly selected to count the number of apoptotic neurons. The apoptosis index $=$ the number of apoptotic cells/total number of cells $\times 100 \%$.

\section{RT-PCR detection of AMPK $m R N A$ expression in rat liver tissue}

The liver tissue samples were ground into powder in liquid nitrogen, and total RNA was then extracted. According to the instructions of the RT-PCR kit (K1003S), $0.5 \mu \mathrm{g}$ of the total tissue RNA and $0.5 \mu \mathrm{g}$ of random primers (synthesized by Invitrogen Inc.) were used for the reverse transcription of cDNA. For AMPK, the upstream primer was 5'-CGA AGC CAG AGC AAA CCA TAC-3', the downstream primer was 5'-TAG CTC CGA TTG TCA ACCAG-3', and the amplified product was $176 \mathrm{bp}$ long; for $\beta$-actin, the upstream primer was $5^{\prime}$ GAG GGA AAT CGT GCG TGAC-3', the downstream primer was 5'-CTG GAA GGT GGA CAG TGAG-3', and the amplified product was $445 \mathrm{bp}$ long. The conditions of the PCR reaction were pre-denaturation at $94^{\circ}$ for 2 minutes, denaturation at $94^{\circ}$ for 30 seconds, annealing at $61^{\circ}$ for 30 seconds, extension at $72^{\circ}$ for 30 seconds for 32 cycles, and extension at $72^{\circ}$ for 2 minutes. Next, $10 \mu \mathrm{L}$ of the PCR amplification products were loaded into ethidium bromide (EB)-containing 1.5\% agarose gel for electrophoresis, and the results were displayed with an automatic digital imaging system. Gene Tools from Syn Gene was used to analyze the optical density of the bands and to calculate the ratio of the optical density between AMPK and the internal reference $\beta$-actin, which represented the relative expression of XIAP mRNA. Each sample was run in triplicate, and the average value was used for the final analysis.

\section{KARGER}




\section{Cellular Physiology Cell Physiol Biochem 2016;38:609-618

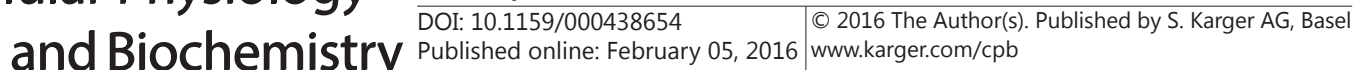 \\ Xia et al.: The Effect of Hippocampal Cognitive Impairment}

Western blot analysis of the expression of AMPK $\alpha$ and phosphorylated AMPK $\alpha$ ( $P A M P K \alpha$ ) in liver tissue

The liver tissue samples were ground into powder in liquid nitrogen, and $100 \mu \mathrm{L}$ of radio immunoprecipitation assay (RIPA) lysis buffer was then added to approximately $10 \mathrm{mg}$ of tissue powder followed by centrifugation at $12,000 \mathrm{rpm}$ and $4^{\circ}$ for 10 minutes. The supernatant was collected for SDSPAGE electrophoresis to separate the proteins; $20 \mu \mathrm{g}$ of the protein samples were loaded into the gel, and after electrophoresis, the proteins were transferred to a polyvinylidene fluoride (PVDF) membrane ( $90 \mathrm{~mA}$, 1.5 hours) followed by washing with tris-buffered saline and tween 20 mixture (TBST) 3 times (10 minutes/ wash). The PVDF membrane was then incubated with an anti-AMPK primary antibody (1:400) and placed on a slow shaker at $4^{\circ}$ overnight. After the TBST wash (3 times, 10 minutes/wash), the membrane was incubated with an HRP-labeled secondary antibody $(1: 5,000)$ on a slow shaker at room temperature for 1 hour. According to the instructions of the ECL kit (Thermo Fisher Scientific Inc.), the PVDF membrane was incubated with the chemiluminescent substrate and then exposed to X-rays. An automatic digital gel imaging system was used to determine and analyze the optical density of the western blot bands. The grayscale value of the target protein was divided by the gray-scale value of internal reference $\beta$-actin, and the result indicated the relative content of the target protein in the sample.

Statistical analysis

The SPSS17.0 software was used for the statistical analysis. The measurement data were expressed as the arithmetic mean \pm standard deviation $(\bar{x} \pm s$ ), and one-way analysis of variance (ANOVA) was performed for inter-group comparisons. $P<0.05$ was considered statistically significant.

\section{Results}

Glucose and lipids levels in rats

TAT-XIAP fusion protein, PBS and XIAP antisense oligonucleotides were injected into the right lateral ventricles of rats with cognitive impairment. The rats were raised for 4 additional weeks, and the results from the automatic biochemical analyzer showed that the levels of FPG, TG, TC, and LDL were significantly higher in the model group relative to the blank group $(P<0.05)$. The blood glucose and lipid levels were significantly lower in the TATXIAP group and significantly higher in the ASODN group than in the model group. The results showed no significant difference in the HDL level between the groups $(P>0.05)$ (Table 1).

\section{Hippocampal neuron apoptosis in rats}

A TUNEL assay was performed to detect hippocampal neuron apoptosis in the rats and showed that apoptotic neuron nuclei were stained with varying shades of brown or yellow. A significant difference in neuron apoptosis was observed between the groups, especially in the hippocampal CA1 region. The results showed that the apoptosis index was $68.44 \pm 4.31 \%$ for the hippocampal CA1 neurons in the model group. Only isolated apoptotic neurons with light staining were observed in the CA1 region of the blank group, and the apoptosis index was $13.21 \pm 2.30 \%$; the apoptosis index of the CA1 region was $56.68 \pm 4.77 \%$ in the TAT-XIAP group and $87.51 \pm 6.63 \%$ in the ASODN group $(P<0.01$; Fig. 1$)$.

Table 1. Glucose and lipids levels in rats $(\bar{x} \pm s)(\mathrm{mmol} / \mathrm{L}) .{ }^{\Delta} P<0.05,{ }^{\Delta \Delta} P<0.05$ vs. Blank group; ^ $P<0.05,{ }^{*} P<0.05$ vs. Model group

\begin{tabular}{lcccccc}
\hline Group & No. of rats & FPG & TG & TC & HDL & LDL \\
\hline Blank group & 10 & $4.20 \pm 0.36$ & $0.83 \pm 0.43$ & $1.53 \pm 0.21$ & $0.77 \pm 0.44$ & $0.51 \pm 0.19$ \\
Model group & 10 & $7.74 \pm 1.21^{\Delta \Delta}$ & $2.35 \pm 0.63^{\Delta}$ & $4.85 \pm 0.95^{\Delta \Delta}$ & $0.87 \pm 0.36$ & $2.64 \pm 0.28^{\Delta}$ \\
TAT-XIAP group & 10 & $5.64 \pm 0.72^{\star}$ & $1.70 \pm 0.59^{\star}$ & $3.40 \pm 0.61^{\star}$ & $0.90 \pm 0.28$ & $1.83 \pm 0.20^{\star}$ \\
ASODN group & 10 & $9.90 \pm 0.55^{\star}$ & $3.46 \pm 0.73^{\star}$ & $5.96 \pm 0.85^{\star}$ & $0.83 \pm 0.34$ & $3.77 \pm 0.43^{\star}$ \\
\hline
\end{tabular}


Table 2. Observation of gastrointestinal motility in rats $(\bar{x} \pm s) .{ }^{\Delta} P<0.05,{ }^{\Delta} P<0.01$ vs. Blank group; ${ }^{\wedge} P<0.05,{ }^{\star} P<0.05$ vs. Model group

\begin{tabular}{lcccc}
\hline Group & $\begin{array}{c}\text { No. of } \\
\text { rats }\end{array}$ & $\begin{array}{c}\text { Frequent gastrointestinal } \\
\text { motility }\end{array}$ & $\begin{array}{c}\text { Gastric emptying rate } \\
(\%)\end{array}$ & $\begin{array}{c}\text { Intestinal propulsive rate } \\
(\%)\end{array}$ \\
\hline Blank group & 10 & $5.53 \pm 1.34$ & $73.16 \pm 12.03$ & $78.77 \pm 19.43$ \\
Model group & 10 & $3.87 \pm 0.61^{\Delta}$ & $44.55 \pm 18.23 \Delta \Delta$ & $40.42 \pm 17.80^{\Delta \Delta}$ \\
TAT-XIAP group & 10 & $5.02 \pm 1.08^{\star}$ & $66.70 \pm 15.41^{\star}$ & $66.33 \pm 17.54^{\star}$ \\
ASODN group & 10 & $2.10 \pm 0.66^{\star}$ & $33.46 \pm 14.27^{\star}$ & $28.10 \pm 8.26^{\star}$ \\
\hline
\end{tabular}

Fig. 1. Detection of hippocampal neuron apoptosis in rats with the TUNEL assay. Blank group (A), Model group (B), ASODN group (C), TAT-XIAP group (D), Negative control (E) $(\times 400)$.
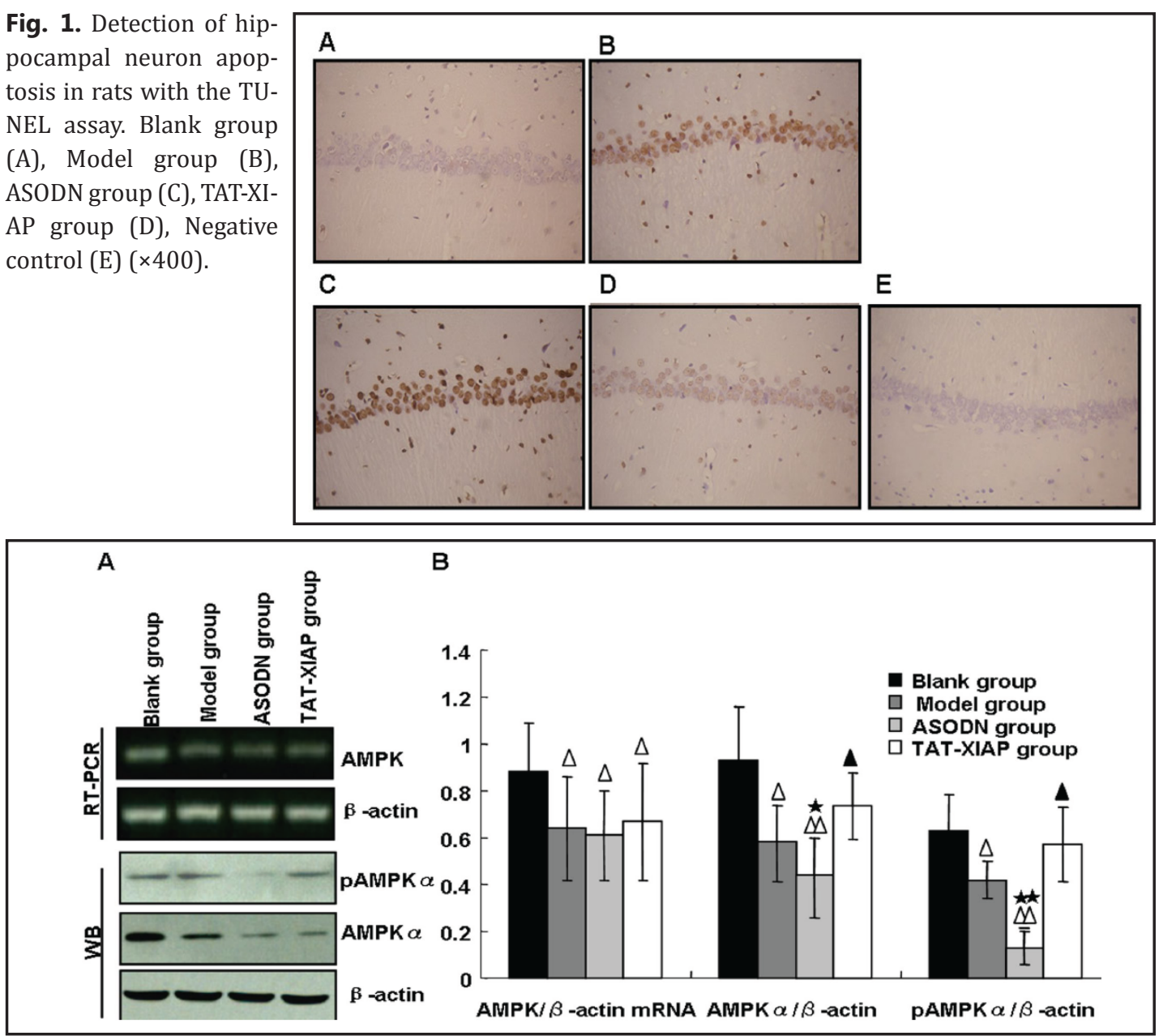

Fig. 2. (A) Expression of AMPK mRNA and protein in four group rats liver by RT-PCR and western blotting, $\beta$-actin was used as a loading control, (B) Histogram showing the protein and mRNA expression levels of AMPK in four group rats liver by RT-PCR and western blotting, $\Delta \mathrm{P}<0.05 \Delta \Delta \mathrm{P}<0.01$ vs. Blank group; $\Delta \mathrm{P}<0.05, * \mathrm{P}<0.05,{ }^{* *} \mathrm{P}<0.01$ vs. Model group.

\section{Observation of gastrointestinal motility in rats}

B-mode ultrasonography showed slow gastrointestinal motility in the model group, the ASODN group and the TAT-XIAP group with less frequent (per time unit) gastrointestinal motility than the blank group. Moreover, the gastrointestinal motility was significantly less frequent in the ASODN group than in the model group and was significantly more frequent in the TAT-XIAP group than in the model group. The gastric emptying rate and intestinal propulsive rate were significantly lower in the model group than in the blank group. Compared with the model group, the gastric emptying rate and intestinal propulsive rate

\section{KARGER}




\section{Cellular Physiology Cell Physiol Biochem 2016;38:609-618 \\ \begin{tabular}{ll|l} 
and Biochemistry Published online: February 05, 2016 & $\begin{array}{l}\text { (c) } 2016 \text { The Author(s). Published by S. Karger AG, Basel } \\
\text { www.karger.com/cpb }\end{array}$ \\
\hline
\end{tabular} \\ Xia et al.: The Effect of Hippocampal Cognitive Impairment}

were significantly lower in the ASODN group and significantly higher in the TAT-XIAP group (Table 2).

AMPK expression in rat liver

TAT-XIAP fusion protein, PBS and XIAP antisense oligonucleotides were injected into the right lateral ventricles of rats. Four weeks later, RT-PCR was performed using rat liver tissue. The amplification fragment was $176 \mathrm{bp}$ for AMPK and $445 \mathrm{bp}$ for $\beta$-actin, which correlated with the primers that were used. Compared with the blank group, AMPK mRNA expression was significantly reduced in the model group, the TAT-XIAP group, and the ASODN group $(P$ $<0.05$ ). Compared with the model group, AMPK mRNA expression was higher in the TATXIAP group and was lower in the ASODN group, but the differences were not statistically significant $(P>0.05)$. The western blot analysis showed that the size of the AMPK $\alpha$ subunit was $63 \mathrm{kD}$. Compared with the blank group, the expression of AMPK $\alpha$ and pAMPK $\alpha$ was significantly lower in the model group, the TAT-XIAP group, and the ASODN group $(P<0.05)$. Compared with the model group, AMPK expression was significantly higher in the TAT-XIAP group and was significantly lower in the ASODN group $(P<0.05)$ (Fig. 2).

\section{Discussion}

Hippocampal neuron apoptosis is the main pathological change of many cognitive disorders, such as $\mathrm{AD}[7,8]$. Cysteinyl aspartate specific proteinase (caspase) plays a critical role in apoptosis and mediates apoptosis through many pathways [9-11]. Caspase cascade also activates microglia cells in brain tissue to promote the secretion of neurotoxic cytokines, such as tumor necrosis factor (TNF)- $\alpha$, interleukin-l $\beta$ (IL-1 $\beta$ ), and interferon- $\gamma$ (IFN- $\gamma$ ), and thereby aggravates neuronal injury [12]. Based on the central role of caspase in neuron apoptosis and injury, researchers are focusing on the inhibition of apoptosis and the protection of neurons by inhibiting caspase activity. XIAP is the most potent caspase inhibitor and can prevent apoptosis by binding to and inhibiting caspase-3, 7, 9 and reducing the release of cytochrome $C$ [13]. In addition to inhibiting caspase activity, XIAP also activates the expression of some anti-apoptotic genes through the nuclear factor kappalight-chain-enhancer of activated B cells (NF- $\mathrm{KB}$ ) pathway and thereby inhibits apoptosis; it also reduces ischemia/hypoxia-induced oxidative stress and injury by upregulating the expression of mitochondrial antioxidants (superoxide dismutase, thioredoxin) [14]. XIAP is a macromolecule of $57 \mathrm{kDa}$; thus, it is difficult for it to cross the blood brain barrier, and its role in the central nervous system has been rarely studied. Thus, it is equally important to look for ways to help macromolecules to cross the blood brain barrier. TAT of type I human immunodeficiency virus (HIV-1) is a well-defined protein transduction domain (PTD) with a potent transduction function [15]. TAT-PTD-mediated protein transduction is independent of the receptor and transporter protein or temperature and energy and has little toxicity to the host cells [16]. Moreover, TAT-PTD is easier to use, has more precise regulation of gene expression and higher efficiency relative to the conventional transgenic methods, and can cross the cell membrane; thus, it has become a useful transport vector for gene therapy and the development of effective drugs and novel vaccines. Both in vivo and in vitro experiments have successfully used fusion proteins that were constructed using TAT-PTD to transduce biological macromolecules (such as glial cell-derived neurotrophic factor [GDNF]) into mammalian cells or into the brain via the blood brain barrier [15-17]. The characteristics and advantages of TAT make it possible to study the role of XIAP in the brain by constructing TAT-XIAP, which carries XIAP across the cell membrane and blood brain barrier and into the brain. We investigated whether TAT carries XIAP across the blood brain barrier and into the brain, and the results showed that genetic engineering techniques could be utilized to construct a biologically active TAT-XIAP fusion protein with high expression in vitro. Moreover, the purity of the fusion protein that was eluted from the Ni-NTA affinity chromatography was greater than $90 \%$. The in vitro experiments showed that TAT-XIAP 
significantly prolonged the survival of the primary fetal rat hippocampal neurons, and the in vivo experiments showed that TAT-XIAP crossed the blood brain barrier and entered the brain.

Anatomically, the hippocampus has complex nerve fiber connections with the hypothalamus, brain stem, and amygdala, and stimulation of the hippocampus may induce changes in the heart rate, respiration and blood pressure via the vagus nerve and sympathetic nerves; stimulation of the peripheral vagus nerve also affects the structure and function of the hippocampus [18]. The hypothalamic - pituitary - adrenal axis (HPA axis) is the main efferent pathway of neuroendocrine pathways. The central nervous system regulates the activity of peripheral organs through the HPA axis. Stimulation of the hippocampal CA3 region, dentate gyrus or subiculum may induce a significant decrease in plasma cortisol, and damage to hippocampal structures, such as the subiculum, may lead to increased secretion of glucocorticoids [19]. Forray MI et al. [20] believed that the hippocampus has direct neuronal connections to the hypothalamic paraventricular nucleus (PVN) and can affect the activity of PVN neuroendocrine cells through rich gamma - aminobutyric acids (GABA) ergic neurons in the forebrain brain nucleus of stria terminalis (BNST) and other hypothalamic relay nuclei, which enables the regulation of the HPA axis. Neurotransmitters such as norepinephrine (NE) and neuropeptide Y (NPY) are also involved in the hippocampal regulation of metabolism [21]. Metabolic abnormalities that are observed in isolated AD cases in clinical practice may be the result of hippocampal lesions that affect both cognitive function and energy metabolism. This study showed that 4 weeks after injection, the levels of blood glucose and the lipid panel in rats with cognitive impairment were significantly increased. Experiments that intervened in hippocampal neuron apoptosis showed that the levels of blood glucose and lipids may be regulated by changing the XIAP activity of the injection side (right side) of the hippocampus; the levels of blood glucose and lipids were significantly decreased (still above the normal range) in the TAT-XIAP group where the XIAP activity was up-regulated, while in the ASODN group, XIAP was inactivated, which exacerbated hippocampal neuron apoptosis and resulted in higher levels of blood glucose and lipids via visceral nerve efferent pathways. These results suggest that XIAP played an important role in the survival of hippocampal neurons and that changes in XIAP activity affected the survival of the hippocampal neurons and glucose and lipids metabolism.

The hippocampus regulates gastrointestinal motility via the vagus nerve. The hippocampus contains gastric distension sensitive neurons that receive the afferent signals from mechanical stimulation of the gastric wall. Gastric electrical stimulation excites gastric distension sensitive neurons in the hippocampal CA1 region [22, 23], and microinjection of motilin into the hippocampal CA3 region stimulates gastric motility, which may be mediated by non-adrenergic, non-cholinergic neurons [24]. This study found that gastrointestinal motility was more frequent and the gastric emptying rate and intestinal propulsive rate were higher in the TAT-XIAP group, where apoptosis was inhibited. Opposite results were observed in the ASODN group, which suggests that hippocampal neuron apoptosis had an important regulatory role in gastrointestinal motility. The frequency of gastrointestinal motility was associated with the extent of neuron apoptosis in the hippocampal CA1 region, and more severe neuronal apoptosis was associated with less frequent gastrointestinal motility. AMPK plays an extremely important regulatory role in energy metabolism, and multiple links involved in cell metabolism respond to changes in the state of cellular energy metabolism to maintain the balance of energy supply and demand $[25,26]$. At a lower level of intracellular adenosine triphosphate (ATP), AMPK inhibits the synthesis of glycogen, fat and cholesterol and thereby reduces ATP use. In addition, AMPK promotes fatty acid oxidation and glucose transport, which increases ATP production [27]. AMPK regulates the homeostasis of liver cholesterol through the target protein hydroxy-3-methylglutaryl coenzyme A reductase (HMG-CoA). Additionally, the hypoglycemic drug metformin inhibits liver gluconeogenesis through an AMPK-mediated orphan nuclear receptor small heterodimer partner (SHP) [28], and the specific knockout of liver kinase B1 (LKB1; upstream to AMPK) led to nearly 100\% inactivation of liver AMPK and elevated FPG. Metformin had no impact on the blood glucose 


\section{Cellular Physiology Cell Physiol Biochem 2016;38:609-618

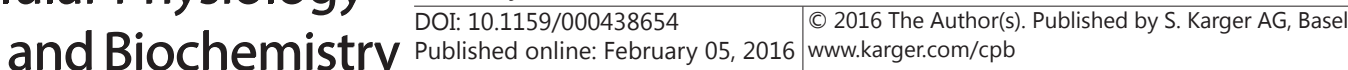 \\ Xia et al.: The Effect of Hippocampal Cognitive Impairment}

level of LKB1 knockout mice, which suggests that the activation of liver AMPK was essential for metformin to exert its hypoglycemic effect [29]. This study showed that AMPK expression in the liver was decreased in the model group, the TAT-XIAP group, and the ASODN group with elevated levels of blood glucose and lipids, which suggests that AMPK may play a role. Compared with the model group, AMPK expression was significantly increased in the TAT-XIAP group and was significantly decreased in the ASODN group $(P<0.05)$, which was presumably related to the neuroendocrine efferent pathways in the hippocampus; however, additional research is needed to investigate the detailed mechanism.

In summary, we believe that hippocampal neuron apoptosis can cause abnormal glucose and lipid metabolism in rats with cognitive impairment, which may be associated with the regulation of gastrointestinal motility and AMPK expression in the liver. Changes in the function of XIAP may also regulate the metabolism of glucose and lipids.

\section{Acknowledgements}

This research was supported in part by The National Natural Science Foundation of China (No. 81260137).

\section{Disclosure Statement}

The authors have declared that no competing interests exist.

\section{References}

1 Petrova M, Prokopenko S, Pronina E, Mozheyko E: Diabetes type 2, hypertension and cognitive dysfunction in middle age women. J Neurol Sci 2010;299:39-41.

2 Manschot SM, Brands AM, van der Grond J, Kessels RP, Algra A, Kappelle LJ, Biessels GJ: Brain magnetic resonance imaging correlates of impaired cognition in patients with type 2 diabetes. Diabetes 2006;55:1106-1113.

3 Biessels GJ, Staekenborg S, Brunner E, Brayne C, Scheltens P: Risk of dementia in diabetes mellitus: a systematic review. Lancet Neurol 2006;5:64-74.

4 Manczak M, Anekonda TS, Henson E, Park BS, Quinn J, Reddy PH:Mitochondria are a direct site of A $\beta$ accumulation in Alzheimer's disease neurons: implications for free radical generation and oxidative damage in disease progression. Hum Mol Genet 2006;15:1437-1449.

5 Keil U, Bonert A, Marques CA, Scherping I, Weyermann J, Strosznajder JB, Müller-Spahn F, Haass C, Czech C, Pradier L, Müller WE, Eckert A: Amyloid $\beta$-induced changes in nitric oxide production and mitochondrial activity lead to apoptosis. J Biol Chem 2004;279:50310-50320.

6 Francis J, Critchley D, Dourish CT, Cooper SJ: Comparisons between the effects of 5-HT and DL-fenfluramine on food intake and gastric emptying in the rat. Pharmacol Biochem Behav 1995;50:581-585.

7 Lu T, Aron L, Zullo J, Pan Y, Kim H, Chen Y, Yang TH, Kim HM, Drake D, Liu XS, Bennett DA, Colaiácovo MP, Yankner BA: REST and stress resistance in ageing and Alzheimer's disease. Nature 2014;507:448-454.

8 Wei HJ, Xu JH, Li MH, Tang JP, Zou W, Zhang P, Wang L, Wang CY, Tang XQ: Hydrogen sulfide inhibits homocysteine-induced endoplasmic reticulum stress and neuronal apoptosis in rat hippocampus via upregulation of the BDNF-TrkB pathway. Acta Pharmacol Sin 2014;35:707-715.

9 Kothakota S, Azuma T, Reinhard C, Klippel A, Tang J, Chu K, McGarry TJ, Kirschner MW, Koths K, Kwiatkowski DJ, Williams LT: Caspase-3-generated fragment of gelsolin: Effector of morphological change in apoptosis. Science 1997;278:294-298.

10 Chen J, Duan Y, Zhang X, Ye Y, Ge B, Chen J: Genistein induces apoptosis by the inactivation of the IGF-1R/pAkt signaling pathway in MCF-7 human breast cancer cells. Food Funct 2015;6:995-1000. 


\section{Cellular Physiology Cell Physiol Biochem 2016;38:609-618 \begin{tabular}{ll|l} 
DOI: 10.1159/000438654 & O 2016 The Author(s). Published by S. Karger AG, Basel \\
and Biochemistry & Published online: February 05, 2016 mwwwkargercom/cpb &
\end{tabular} \\ Xia et al.: The Effect of Hippocampal Cognitive Impairment}

11 Chen J, Lin C, Yong W, Ye Y, Huang Z: Calycosin and genistein induce apoptosis by inactivation of HOTAIR/pAkt signaling pathway in human breast cancer MCF-7 cells. Cell Physiol Biochem 2015;35:722-728.

12 Burguillos MA, Deierborg T, Kavanagh E, Persson A, Hajji N, Garcia-Quintanilla A, Cano J, Brundin P, Englund E, Venero JL, Joseph B: Caspase signalling controls microglia activation and neurotoxicity. Nature 2011;472:319-324.

13 Deveraux QL, Takahashi R, Salvesen GS, Reed JC: X-linked IAP is a direct inhibitor of cell-death proteases. Nature 1997;388:300-304.

14 Aguilar C, Lenoir C, Lambert N, Bègue B, Brousse N, Canioni D, Berrebi D, Roy M, Gérart S, Chapel H, Schwerd T, Siproudhis L, Schäppi M, Al-Ahmari A, Mori M, Yamaide A, Galicier L, Neven B, Routes J, Uhlig HH, Koletzko S, Patel S, Kanegane H, Picard C, Fischer A, Bensussan NC, Ruemmele F, Hugot JP, Latour S: Characterization of Crohn disease in X-linked inhibitor of apoptosis-deficient male patients and female symptomatic carriers. J Allergy Clin Immunol 2014;134:1131-1141.

15 Nemes C, Varga E, Polgar Z, Klincumhom N, Pirity MK, Dinnyes A: Generation of mouse induced pluripotent stem cells by protein transduction. Tissue Eng Part C Methods 2014;20:383-392.

16 Doeppner TR, Dietz GP, El Aanbouri M, Gerber J, Witte OW, Bähr M, Weise J: TAT-Bcl-xL improves survival of neuronal precursor cells in the lesioned striatum after focal cerebral ischemia. Neurobiol Dis 2009;34:8794.

17 Kilic U, Kilic E, Dietz GP, Bähr M: Intravenous TAT-GDNF is protective after focal cerebral ischemia in mice. Stroke 2003;34:1304-1310.

18 Theodore WH, Fisher RS:Brain stimulation for epilepsy. Lancet Neurol 2004;3:111-118.

19 Mueller NK, Dolgas CM, Herman JP: Stressor-selective role of the ventral subiculum in regulation of neuroendocrine stress responses. Endocrinology 2004;145:3763-3768.

20 Forray MI, Gysling K: Role of noradrenergic projections to the bed nucleus of the stria terminalis in the regulation of the hypothalamic-pituitary-adrenal axis. Brain Res Rev 2004;7:145-160.

21 Morishima M, Harada N, Hara S, Sano A, Seno H, Takahashi A, Morita Y, Nakaya Y: Monoamine oxidase A activity and norepinephrine level in hippocampus determine hyperwheel running in SPORTS rats. Neuropsychopharmacology 2006;31:2627-2638.

22 Davidson TL, Kanoski SE, Chan K, Clegg DJ, Benoit SC, Jarrard LE: Hippocampal lesions impair retention of discriminative responding based on energy state cues. Behav Neurosci 2010;124:97-105.

23 Xu L, Sun X, Tang M, Chen JD: Involvement of the hippocampus and neuronal nitric oxide synapse in the gastric electrical stimulation therapy for obesity. Obes Surg 2009;19:475-483.

24 Guan Y, Tang M, Jiang Z, Peeters TL: Excitatory effects of motilin in the hippocampus on gastric motility in rats. Brain Res 2003;984:33-41.

25 Cantó C, Gerhart-Hines Z, Feige JN, Lagouge M, Noriega L, Milne JC, Elliott PJ, Puigserver P, Auwerx J: AMPK regulates energy expenditure by modulating NAD+ metabolism and SIRT1 activity. Nature 2009;458:10561060.

26 Hardie DG. AMPK: positive and negative regulation, and its role in whole-body energy homeostasis. Curr Opin Cell Biol 2014;33:1-7.

27 Hardie DG, Ross FA, Hawley SA. AMPK: a nutrient and energy sensor that maintains energy homeostasis. Nat Rev Mol Cell Biol 2012;13:251-262.

28 Kim YD, Park KG, Lee YS, Park YY, Kim DK, Nedumaran B, Jang WG, Cho WJ, Ha J, Lee IK, Lee CH, Choi HS: Metformin inhibits hepatic gluconeogenesis through AMP-activated protein kinase-dependent regulation of the orphan nuclear receptor SHP. Diabetes 2008;57:306-314.

29 Shaw RJ, Lamia KA, Vasquez D, Koo SH, Bardeesy N, Depinho RA, Montminy M, Cantley LC: The kinase LKB1 mediates glucose homeostasis in liver and therapeutic effects of metformin. Science 2005;310:1642-1646. 